\title{
Organizing Chaos: Iterative Professional Identity Formation Through the Lens of Mask Making
}

\author{
Mark B. Stephens, MD, MS | Joy L. Bowen, MPIA | Erin L. McGinley | Peter Rainey
}

PRiMER. 2020;4:10.

Published: 6/18/2020 | DOI: 10.22454/PRiMER.2020.705788

\section{Abstract}

Introduction: Professional identity formation (PIF) is a sociocultural process through which medical students adopt the professional role of physician. This process is often unscripted and influenced by informal curricular elements. PIF is as important as the acquisition of knowledge and clinical skill in the continuum of medical education.

Methods: Using the ancient art of mask making, we created a process of reflective expression to explicitly examine and formally promote PIF. Students created individual masks to express elements of self in the context of their medical education experiences. Coupled with a narrative reflection describing the mask and the process of mask making, students were challenged to examine and give shape to their evolving sense of professional identity. Using a retrospective pre/post design, we used the mask-making process to examine identity across 4 years of medical school in a cohort of graduating students.

Results: The masks and accompanying narratives showed themes of moving from anxiety and uncertainty at matriculation to a more calm and focused state at the time of graduation. Other themes included the ability to organize complex material and the accumulation of a broad fund of knowledge. Students found the maskmaking experience to be introspective and enjoyable.

Discussion: Mask making is both a product (mask) and process (creation). As such, mask making is an innovative strategy to examine PIF within individuals and across time. Organization, focus, and selfunderstanding were common themes of professional growth.

\section{Introduction}

Medical schools must provide students with the scientific knowledge and clinical skills to practice medicine while simultaneously helping them to develop a healthy sense of professional identity. ${ }^{1}$ In this context, professional identity formation (PIF) is as important as the acquisition of knowledge and clinical skills in the overall mission of medical education. ${ }^{2}$ The development of a healthy professional identity requires self-awareness and a regular habit of reflective practice. ${ }^{3}$ Like the process of learning itself, identity formation is a complex phenomenon ${ }^{4}$ that is shaped by multiple factors. ${ }^{5,6}$ Meaningful professional learning occurs when individuals engage in constructive activities within a specific community of practice. ${ }^{7}$ As an exemplar community of practice, medicine has a unique set of symbols, language, traditions, and rites of passage that entering students must navigate to be accepted as a full-fledged member of the profession. This social and cognitive development is foundational to PIF for medical students.

To date, most reflective activities in medical education have used writing activities as the primary instructional tool. ${ }^{8}$ 
However, the exclusive use of a narrative focus may limit some students' ability to convey thoughts or experiences, particularly for those who struggle to write or fully express their perspectives through the written word. To complement this, we have reintroduced the art of mask making as a vehicle for self-reflection and expression. ${ }^{9}$ Masks symbolize the social and relational nature of the human experience. ${ }^{10}$ Masks are used for exploration (Who am I?); transformation (Who am I becoming?); and understanding (Who do I want to be?). ${ }^{11}$ Mask making represents a novel modality to examine PIF as students shape new personas during their medical education. Art gives representation to the invisible and voice to the otherwise silent self. ${ }^{12}$ Masks explore sociocultural perspectives of transition, environment, and learning to visually convey stories and stimulate emotions. ${ }^{13}$ Themes such as role strain, burnout, identity dissonance, ${ }^{14}$ and reclamation of authentic elements of personal identity ${ }^{15}$ have been identified in our prior work using mask making to examine medical student identity formation. The goal of the present study was to use mask-making to examine self-described changes in identity from the first through the fourth year of medical school in a small cohort of graduating students.

\section{Methods}

We used a conceptual framework for arts-based activities in medical education, ${ }^{16}$ proposing that learners should (1) embrace the ambiguous and complex nature of the arts to (2) engage in an aesthetic, reflective activity to (3) construct new meanings that they then (4) translate to medical practice. Our qualitative design used mask making and an accompanying narrative to explore self-reported changes in professional identity in a small cohort of medical students over time. ${ }^{9}$ Following institutional review board approval and student consent, graduating students from the Penn State College of Medicine University Park Regional Campus were invited to participate in the study. We used a retrospective pre/postdesign whereby students were asked to create one mask to express a recollection of their sense of professional self during their first year of medical school (Mask 1) and a second mask representing their sense of professional self at the time of graduation (Mask 2). To enrich the qualitative interpretation of the masks, students created a reflective narrative to describe the meaning of each of their masks.

A rubric was created using principles of visual rhetoric ${ }^{17}$ and the Listening Guide ${ }^{18,19}$ to examine the masks and essays. As the first step, each author analyzed the masks and narratives independently to identify general themes. Next, the authors came together to compare and consolidate findings. ${ }^{20}$ We used grounded theory (a qualitative methodology that applies inductive reasoning $)^{21,22}$ to reach group consensus. Once the project team reached data saturation, where no new themes from coding emerged from conversations about the data, analytic consensus was agreed upon. ${ }^{23}$ We retained individual and summary notes as an audit trail for confirmability of findings.

The basic study design inherently satisfies the first element of the adopted framework. ${ }^{16}$ To engage with the arts, we used the creative process of mask making coupled with the reflective narrative. Mask making is a medium that allows for metaphorical representation. The process of mask making is subjective and complex, requiring visual and tactile engagement. Finally, participants were challenged to intentionally reflect on their experiences (and emotions relating to those experiences) during medical school; to consider what lessons they learned that they planned to apply to residency, and to use the the mask as a vehicle to interpret and express themselves. Coupled with the reflective narrative, this part of the study design satisfied the final elements of the art-based medical education framework: construction of new meanings and translation to medical practice.

\section{Results}

Five of $12(42 \%)$ graduating students created both of the masks and accompanying narrative. The composition of the masks varied in color, spatial organization, and expressive content. Visual thematic analysis of the masks suggested the use of color to convey emotion and space to convey organization (Table 1). The accompanying narratives reinforced common themes of organization, calm, and expanded knowledge. 


\section{Construction of New Meaning and Translation to Medical Practice}

\section{Theme 1: Organization}

One participant contrasted white space and irregular lines with warm and cool tones to visually represent the pressures of medical school pushing them to become more organized. Another participant referred to newly learned approaches in problem solving and patient interaction to promote organizational skills. A third participant created a sense of "organized chaos" in their mask as an expression of both increased knowledge and an improved ability to organize the complexity of medicine.

\section{Theme 2: Calm}

One participant used a bright window surrounded by darkness and black bars to represent a state of anxiety. This contrasted with wisps of white color signifying pure, free thoughts at the time of graduation. A second participant used the color blue to represent an increased sense of calm at the end of medical school. A third participant also used bright colors to symbolize optimism about the future and a feeling of peace.

\section{Theme 3: Knowledge and Self-awareness}

One participant contrasted bright, fresh colors (fertile ground symbolizing a readiness to learn) with rich brown soil dotted with jewels to represent significant knowledge acquisition. Another participant reflected that in addition to increased knowledge, they felt a significant maturation and improved self-awareness. A third participant created a simple mask to visually represent their initially simplistic understanding of the practice of medicine. Diverse color and complex patterns were used on the second mask to represent the size and complexity of medical knowledge gained, and to celebrate diversity. A fourth participant highlighted how the challenges of medical school rendered significant changes to self through a reexamination of preconceptions and an evaluation of core beliefs.

\section{Conclusion}

To date, several important ideas have emerged from the existing literature regarding mask making and PIF. Our previous work identified common themes of role strain and identity dissonance in third-year students. ${ }^{14} \mathrm{~A}$ second study of third-year students highlighted reclamation of personal identity and integration of professional identity into an overall sense of self. ${ }^{15}$ The present study adds the perspective of students sharing from first- and fourth-year perspectives. The strongest themes to emerge were a sense of calm and organization over time, and increased selfawareness, moving from "fictitious depth" and "discombobulated chaos" at the beginning of medical school to organization, focus, and improved knowledge at graduation. This represents an important finding in the context of professional identity as students transition from medical school to residency. This builds on prior findings by providing further insight on the use of mask making as art form to construct new meaning and to translate this to medical practice. Students expressed an increased sense of self-awareness and appeared ready to apply their expanded fund of knowledge in a focused way at the next level of their ongoing medical education.

This study has several limitations. First, the sample size was small. It is possible that the five students who chose to participate did so because they enjoy the arts and/or art-making processes. This introduces an element of selfselection bias into the study. In addition, a small sample size from a single institution makes generalizability challenging. However, as the overall study design was intentionally qualitative and exploratory in nature, as the goal was hypothesis generation rather than hypothesis testing.

Recognizing that individuals view their present selves differently than their past or future selves, ${ }^{4}$ mask making is a novel vehicle ${ }^{24}$ to examine identity formation over time. Mask-making allows students to translate thoughts and emotions onto the mask, combining form and content to evoke visual and emotional reactions. Students can explore distressing experiences, decrease shame or fear of exposure, and break through layers of psychological confinement and/or internalization of emotions. ${ }^{25}$ Adding mask making as another arts-based vehicle for selfexploration and reflective expression potentially helps students to attain a more comprehensive sense of professional identity. Next steps for future research include comparing the effectiveness of different modalities of 
reflection in the context of PIF. To do this, we plan to introduce structured interviews to better understand student descriptions of their lived experiences of professional identity formation.

\section{Tables and Figures}

Table 1: Mask Making: Products and Process

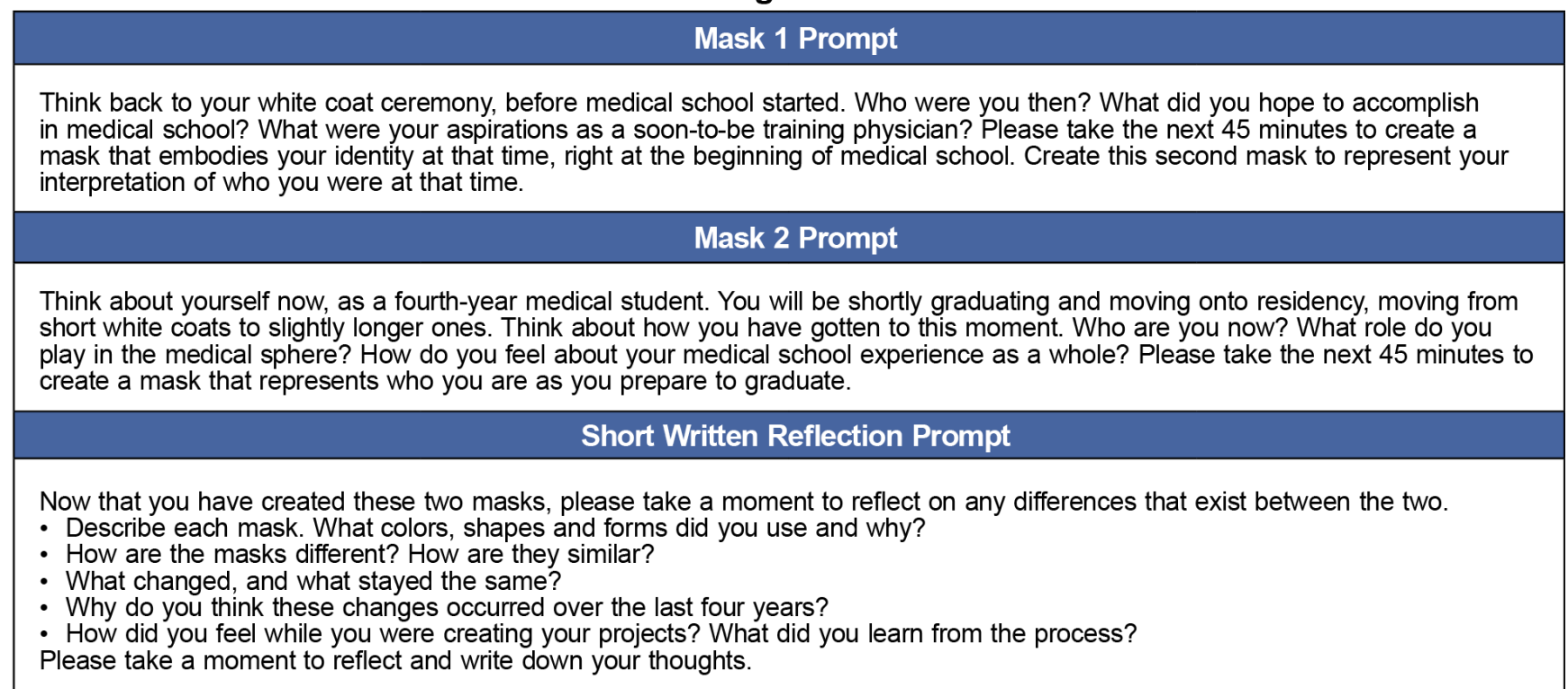

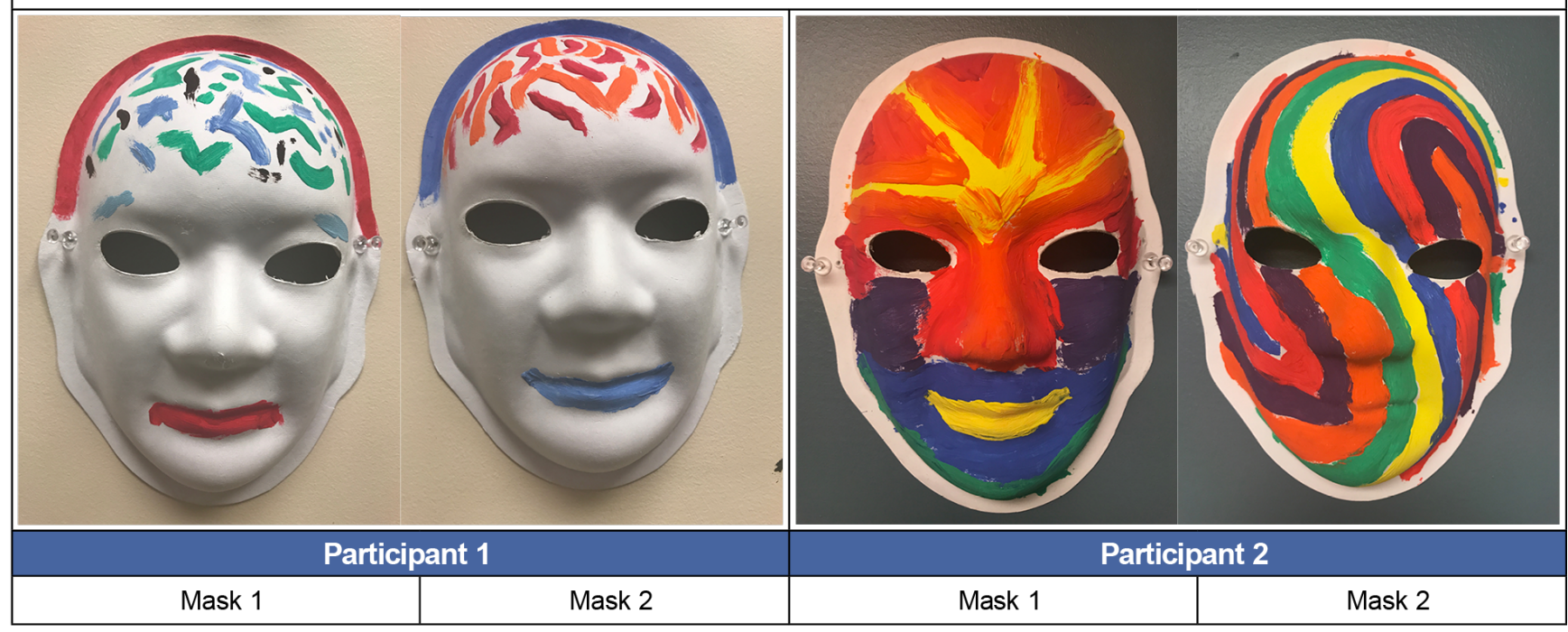

(Table continued on next page) 
(Table 1: Continued)

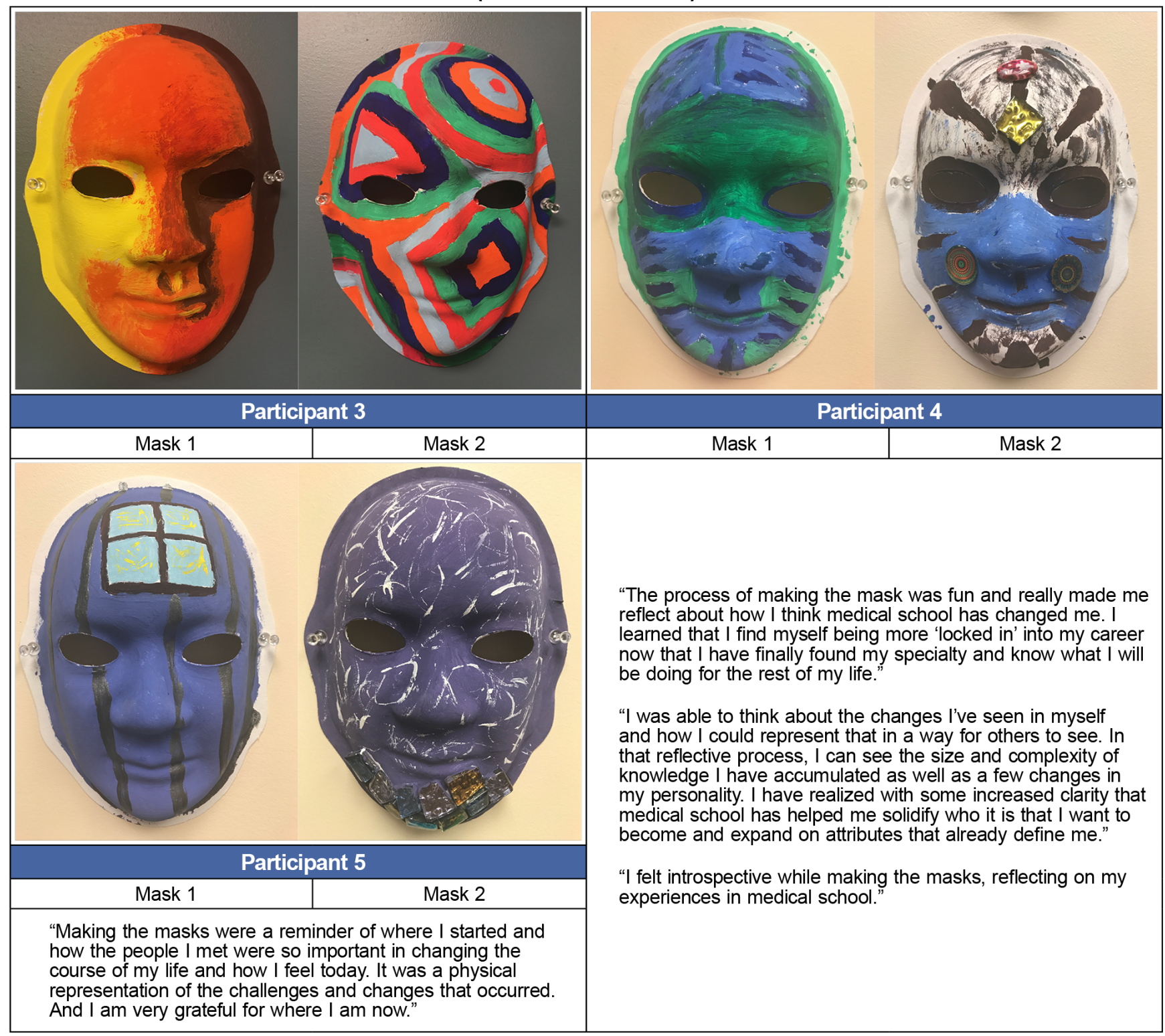

\section{Acknowledgments}

Ethical Approval: This research was conducted in accordance with the Declaration of Helsinki. There was no identified risk of harm to participants, efforts to ensure anonymity of participants were taken, and consent of all participants was obtained.

\section{Corresponding Author}

Mark B. Stephens, MD, MS

1850 E. Park Ave, Suite 304, State College, PA 16803. 910-238-6101

Mstephens3@pennstatehealth.psu.edu

\section{Author Affiliations}

Mark B. Stephens, MD, MS - Penn State University College of Medicine, State College, PA

Joy L. Bowen, MPIA - Department of Family and Community Medicine, Penn State College of Medicine 
Erin L. McGinley - Department of Family and Community Medicine, Penn State College of Medicine

Peter Rainey - Penn State Health Family and Community Medicine Residency at Mount Nittany Medical Center

\section{References}

1. Cruess RL, Cruess SR, Boudreau JD, Snell L, Steinert Y. Reframing medical education to support professional identity formation. Acad Med. 2014;89(11):1446-1451. https://doi.org/10.1097/ACM.0000000000000427

2. Cooke M, Irby DM, MacLeod A. Educating physicians: A call for reform of medical school and residency. San Francisco, CA: Josey Bass; 2010.

3. Mann K, Gordon J, MacLeod A. Reflection and reflective practice in health professions education: a systematic review. Adv Health Sci Educ Theory Pract. 2009;14(4):595-621. https://doi.org/10.1007 /s10459-007-9090-2

4. Vygotsky LS. Mind in society: The development of higher psychological processes. Cambridge, MA: Harvard University Press; 1978.

5. Philibert I, Elsey E, Fleming S, Razack S. Learning and professional acculturation through work: examining the clinical learning environment through the sociocultural lens. Med Teach. 2019;41(4):398-402. https://doi.org $/ 10.1080 / 0142159 \times .2019 .1567912$

6. Egan T, Jaye C. Communities of clinical practice: the social organization of clinical learning. Health (London). 2009;13(1):107-125. https://doi.org/10.1177/1363459308097363

7. Lave J, Wenger E. Situated learning: Legitimate peripheral participation. Cambridge, England: University of Cambridge Press; 1991. https://doi.org/10.1017/CB09780511815355

8. Ng SL, Kinsella EA, Friesen F, Hodges B. Reclaiming a theoretical orientation to reflection in medical education research: a critical narrative review. Med Educ. 2015;49(5):461-475. https://doi.org/10.1111/medu.12680

9. Stephens MB, Bader KS, Myers KR, Walker MS, Varpio L. Examining professional identity formation through the ancient art of mask-making. J Gen Intern Med. 2019;34(7):1113-1115. https://doi.org/10.1007 /s11606-019-04954-3

10. Roy C, Eales J. Masks \& mirrors: from autobiographical reflection to unmasking interdisciplinary collaboration. Reflective Pract. 2010;11(4):433-450. https://doi.org/10.1080/14623943.2010.505716

11. Bentley LL. Investigating the use of creative mask-making as a means to explore professional identity of doctoral psychology students. 2016. Dissertations \& Theses. 319. Retrieved from http://aura.antioch.edu /etds/319

12. Trepal-Wollenzier HC, Wester KL. The use of masks in counseling: Creating reflective space. J of Clin Activities. Assignments \& Handouts in Psychother Pract. 2002;2(2):123-130.

13. Edson G. Masks and masking: Faces of tradition and belief worldwide. Jefferson, NC: McFarland; 2009.

14. Joseph K, Bader K, Wilson S, et al. Unmasking identity dissonance: Exploring medical students' professional identity formation through mask making. Perspect Med Ed. 2017; 6(2): 99-107.

15. Shapiro J, Youm J, Heare M, et al. Medical students' efforts to integrate and/or reclaim authentic identity: insights from a mask-making exercise. J Med Humanit. 2018;39(4):483-501. https://doi.org/10.1007 /s10912-018-9534-0

16. Haidet $P$, Jarecke J, Adams NE, et al. A guiding framework to maximise the power of the arts in medical education: a systematic review and metasynthesis. Med Educ. 2016;50(3):320-331. https://doi.org/10.1111 /medu. 12925

17. Kress G, Van Leeuwen T. Reading images: The grammar of visual design. London, England/New York, NY: Routledge; 2006. https://doi.org/10.4324/9780203619728

18. Gilligan C, Eddy J. Listening as a path to psychological discovery: an introduction to the Listening Guide. Perspect Med Educ. 2017;6(2):76-81. https://doi.org/10.1007/s40037-017-0335-3

19. Varpio L, Grassau P, Hall P. Looking and listening for learning in arts- and humanities-based creations. Med Educ. 2017;51(2):136-145. https://doi.org/10.1111/medu.13125

20. Mays N, Pope C. Quality in qualitative research. In: Pope C, Mays N, eds. Qualitative research in health care. 2nd ed. London: BMJ Books; 2000:89-101.

21. Charmaz K. Constructing grounded theory: a practical guide through qualitative analysis. Thousand Oaks, CA: 
SAGE; 2006.

22. Chun Tie Y, Birks M, Francis K. Grounded theory research: A design framework for novice researchers. SAGE Open Med. 2019;7:2050312118822927. https://doi.org/10.1177/2050312118822927

23. Bradley EH, Curry LA, Devers KJ. Qualitative data analysis for health services research: developing taxonomy, themes, and theory. Health Serv Res. 2007;42(4):1758-1772. https://doi.org/10.1111 /j.1475-6773.2006.00684.x

24. Cox S, Brett-MacLean P, Corneya CA. "My turbinado sugar": Art-making, well-being and professional identity in medical education. Arts Health. 2016;8(1):65-81. https://doi.org/10.1080/17533015.2015.1037318

25. Goldie J. The formation of professional identity in medical students: considerations for educators. Med Teach. 2012;34(9):e641-e648. https://doi.org/10.3109/0142159X.2012.687476

Copyright $\odot 2020$ by the Society of Teachers of Family Medicine 